\title{
AN EXAMPLE OF NON-KELLER MAPPING
}

\author{
Edyta Pawlak $^{1}$, Sylwia Lara-Dziembek ${ }^{1}$, Grzegorz Biernat ${ }^{1}$ \\ Magdalena Woźniakowska \\ ${ }^{I}$ Institute of Mathematics, Czestochowa University of Technology \\ Częstochowa, Poland \\ ${ }^{2}$ Faculty of Mathematics and Computer Science, University of Lodz \\ Łódź, Poland \\ edyta.pawlak@im.pcz.pl,sylwia.lara@im.pcz.pl,grzegorz.biernat@im.pcz.pl \\ magdalena_wozniakowska@wp.pl
}

\begin{abstract}
In the paper a nontrivial example of non-Keller mapping is considered. It is shown that the Jacobian of rare mapping, having one zero at infinity, being constant must vanish.
\end{abstract}

Keywords: Jacobian, zero at infinity, rare mappings, Keller mappings

\section{Introduction}

In this paper we consider the rare polynomial mappings of two complex variables. We study the mappings having one zero at infinity [1-3]. We prove that if the Jacobian of this mapping is constant, it must be zero. The work concerns the problems related to the Keller mappings [4-6]. Recall that the Keller mapping is a polynomial mapping $F: \mathbf{C}^{2} \rightarrow \mathbf{C}^{2}$ satisfies the condition Jac $F=$ const $\neq 0$. In this work, non-Keller mapping are those whose the Jacobian being constant must vanish.

\section{The rare mappings}

Let $f, g: \mathbf{C}^{2} \rightarrow \mathbf{C}$ be the complex polynomials of degrees $2 k+1$ and $2 k$, consequently, and having one zero at infinity. We consider the rare mappings, i.e. the mappings, whose number of zero forms following the leading form is not more than $k-1$. In our case, the mapping has exactly $k-1$ zero forms.

Therefore, we can write

$$
f=X^{2 k+1}+\underbrace{0+\ldots+0}_{k-1 \text { zeros }}+f_{k+1}+\ldots+f_{1}
$$


and

$$
g=X^{2 k}+\underbrace{0+\ldots+0}_{k-1 \text { zeros }}+g_{k}+\ldots+g_{1}
$$

where $k \geq 2$ and $f_{i}, g_{j} \in \mathbf{C}[X, Y]$ are the forms indicated degrees.

\section{Basic lemma}

At the beginning let us provide the following property:

Property. If $X^{2 k-1} \mid g_{k}^{2}$, then $g_{k}=B_{k} X^{k}, k \geq 1$.

Proof.

Must be

$$
g_{k}=A_{k} X^{k}+B_{k} X^{k-1} Y, k \geq 1
$$

Therefore

$$
g_{k}^{2}=A_{k}^{2} X^{2 k}+2 A_{k} B_{k} X^{2 k-1} Y+B_{k}^{2} X^{2 k-2} Y^{2}
$$

and since $X^{2 k-1} \mid g_{k}^{2}$, so $B_{k}=0$.

We assume

$$
\operatorname{Jac}(f, g)=\operatorname{Jac}\left(f_{1}, g_{1}\right)=\text { const }
$$

Let's prove that $\operatorname{Jac}(f, g)=0$.

Lemma. Really, with the given assumptions, we have $\operatorname{Jac}(f, g)=0$.

Proof. Let

$$
\begin{aligned}
f & =X^{2 k+1}+\underbrace{{ }^{k+2)}\left|0+{ }^{k+3)}\right| 0+\ldots+0}_{k-1 \text { zeros }}+\left.f_{k+1}\right|^{1)}+\left.f_{k}\right|^{2)}+\left.f_{k-1}\right|^{3)}+\ldots+ \\
& +\left.f_{4}\right|^{k-2)}+{ }^{3 k-1)}\left|f_{3}\right|^{k-1)}+\left.f_{2}\right|^{k)}+\left.f_{1}\right|^{k+1)}
\end{aligned}
$$




$$
\begin{aligned}
g & =X^{2 k}+\underbrace{{ }^{k+2)}\left|0+{ }^{k+3)}\right| 0+\ldots+0}_{k-1 \text { zeros }}+\left.g_{k}\right|^{1)}+\left.g_{k-1}\right|^{2)}+\left.g_{k-2}\right|^{3)}+\ldots+ \\
& +\left.g_{3}\right|^{k-2)}+{ }^{3 k-1)}\left|g_{2}\right|^{k-1)}+\left.g_{1}\right|^{k)}+\left.0\right|^{k+1)}
\end{aligned}
$$

Since the Jacobian is constant, we have in sequence

$$
\text { 1) } \operatorname{Jac}\left(X^{2 k+1}, g_{k}\right)=\operatorname{Jac}\left(X^{2 k}, f_{k+1}\right)
$$

so

$$
\frac{2 k+1}{2 k} X g_{k}+a_{k+1} X^{k+1}=f_{k+1}
$$

and next

$$
\text { 2) } \operatorname{Jac}\left(X^{2 k+1}, g_{k-1}\right)=\operatorname{Jac}\left(X^{2 k}, f_{k}\right)
$$

therefore

$$
\frac{2 k+1}{2 k} X g_{k-1}+a_{k} X^{k}=f_{k}
$$

and

$$
\text { 3) } \operatorname{Jac}\left(X^{2 k+1}, g_{k-2}\right)=\operatorname{Jac}\left(X^{2 k}, f_{k-1}\right)
$$

however

$$
\frac{2 k+1}{2 k} X g_{k-2}+a_{k-1} X^{k-1}=f_{k-1}
$$

etc.

Finally in the step $k$ ) we have

$$
\text { k) } \operatorname{Jac}\left(X^{2 k+1}, g_{1}\right)=\operatorname{Jac}\left(X^{2 k}, f_{2}\right)
$$

so

$$
\frac{2 k+1}{2 k} X g_{1}+a_{2} X^{2}=f_{2}
$$


Now, an important step

$$
k+1) \underbrace{\operatorname{Jac}\left(f_{k+1}, g_{k}\right)}_{1^{\circ}}=\operatorname{Jac}\left(X^{2 k}, f_{1}\right)
$$

where according to the formula (9) we have

$$
\begin{aligned}
1^{\circ} & =\operatorname{Jac}\left(\frac{2 k+1}{2 k} X g_{k}+a_{k+1} X^{k+1}, g_{k}\right)= \\
& =\frac{2 k+1}{2 k} g_{k} \operatorname{Jac}\left(X, g_{k}\right)+(k+1) a_{k+1} X^{k} \operatorname{Jac}\left(X, g_{k}\right)
\end{aligned}
$$

Returning to the formula (16) we obtain

$$
\frac{2 k+1}{2 k} g_{k} \operatorname{Jac}\left(X, g_{k}\right)+(k+1) a_{k+1} X^{k} \operatorname{Jac}\left(X, g_{k}\right)=2 k X^{2 k-1} \operatorname{Jac}\left(X, f_{1}\right)
$$

then

$$
\frac{2 k+1}{4 k} g_{k}^{2}+(k+1) a_{k+1} X^{k} g_{k}+a_{1} X^{2 k}=2 k X^{2 k-1} f_{1}
$$

This means that

$$
X^{2 k-1} \mid g_{k}^{2}
$$

so, according to the Property, we obtain

$$
g_{k}=B_{k} X^{k}, \quad f_{k+1}=A_{k+1} X^{k+1}
$$

and reusing the formula (19) we have

$$
f_{1}=A_{1} X
$$

In the next step we receive

$$
k+2) \underbrace{\operatorname{Jac}\left(f_{k+1}, g_{k-1}\right)}_{1^{\circ}}+\underbrace{\operatorname{Jac}\left(f_{k}, g_{k}\right)}_{2^{\circ}}=0
$$

where according to the formulas (21) and (11) we get

$$
1^{\circ}=\operatorname{Jac}\left(A_{k+1} X^{k+1}, g_{k-1}\right)=(k+1) A_{k+1} X^{k} \operatorname{Jac}\left(X, g_{k-1}\right)
$$


and

$$
\begin{aligned}
2^{\circ} & =\operatorname{Jac}\left(\frac{2 k+1}{2 k} X g_{k-1}+a_{k} X^{k}, B_{2 k} X^{k}\right)= \\
& =-\frac{2 k+1}{2 k} B_{2 k} X^{k} \operatorname{Jac}\left(X, g_{k-1}\right)
\end{aligned}
$$

Returning to the formula (23) we have

$$
\left[(k+1) A_{k+1}-\frac{2 k+1}{2 k} B_{2 k}\right] \operatorname{Jac}\left(X, g_{k-1}\right)=0
$$

Let's

$$
C_{k}=(k+1) A_{k+1}-\frac{2 k+1}{2 k} B_{2 k}
$$

We consider two cases:

(I) $C_{k} \neq 0$

Then

$$
g_{k-1}=B_{k-1} X^{k-1}, \quad f_{k}=A_{k} X^{k}
$$

In the next steps we receive in sequence

$$
k+3) \operatorname{Jac}\left(f_{k+1}, g_{k-2}\right)+\operatorname{Jac}\left(f_{k}, g_{k-1}\right)+\operatorname{Jac}\left(f_{k-1}, g_{k}\right)=0
$$

so

$$
g_{k-2}=B_{k-2} X^{k-2}, \quad f_{k-1}=A_{k-1} X^{k-1}
$$

etc.

Finally, in the step $2 k$ ) we have

$$
g_{1}=B_{1} X, f_{2}=A_{2} X^{2}
$$

Thus, according to the formula (22) we obtain

$$
\operatorname{Jac}\left(f_{1}, g_{1}\right)=\operatorname{Jac}\left(A_{1} X, B_{1} X\right)=0
$$

what concludes the proof in the first case. 
(II) $C_{k}=0$

Then in the step $k+3$ ) we get

$$
k+3) \operatorname{Jac}\left(f_{k+1}, g_{k-2}\right)+\operatorname{Jac}\left(f_{k}, g_{k-1}\right)+\operatorname{Jac}\left(f_{k-1}, g_{k}\right)=0
$$

So using the formulas (11), (13), (21) and (28) we have

$$
\begin{aligned}
& \operatorname{Jac}\left(A_{k+1} X^{k+1}, g_{k-2}\right)+\operatorname{Jac}\left(\frac{2 k+1}{2 k} X g_{k-1}+a_{k} X^{k}, g_{k-1}\right)+ \\
& +\operatorname{Jac}\left(\frac{2 k+1}{2 k} X g_{k-2}+a_{k-1} X^{k-1}, B_{k} X^{k}\right)=0
\end{aligned}
$$

hence

$$
\frac{2 k+1}{2 k} g_{k-1} \operatorname{Jac}\left(X, g_{k-1}\right)+k a_{k} X^{k-1} \operatorname{Jac}\left(X, g_{k-1}\right)=0
$$

and

$$
\left(\frac{2 k+1}{2 k} g_{k-1}, k a_{k} X^{k-1}\right) \operatorname{Jac}\left(X, g_{k-1}\right)=0
$$

therefore

$$
\begin{aligned}
& C_{k} X^{k} \operatorname{Jac}\left(X, g_{k-2}\right)+\frac{2 k+1}{2 k} g_{k-1} \operatorname{Jac}\left(X, g_{k-1}\right)+ \\
& +k a_{k} X^{k-1} \operatorname{Jac}\left(X, g_{k-1}\right)=0
\end{aligned}
$$

Because $C_{k}=0$, then

$$
\left(\frac{2 k+1}{2 k} g_{k-1}+k a_{k} X^{k-1}\right) \operatorname{Jac}\left(X, g_{k-1}\right)=0
$$

Consequently

$$
g_{k-1}=\widehat{B}_{k-1} X^{k-1}, \quad f_{k}=\widehat{A}_{k} X^{k}
$$

In the following steps, depending on the obtained values of the coefficients (non-zero or zero), we obtain the successive values of the forms

$$
g_{l-1}=\widehat{B}_{l-1} X^{l-1}, \quad f_{l}=\widehat{A}_{l} X^{l}, \quad l \leq k-1
$$


When all the subsequent coefficients are zero, in step $3 k-1)$ we have

$$
g_{1}=\widehat{B}_{1} X, \quad f_{2}=\widehat{A}_{2} X^{2}
$$

Thus, according to the formula (22) we obtain

$$
\operatorname{Jac}\left(f_{1}, g_{1}\right)=\operatorname{Jac}\left(\widehat{A}_{1} X, \widehat{B}_{1} X\right)=0
$$

which ends the proof in the second case.

\section{Conclusion}

In this article we consider the "frontier" case of rare and non-Keller mapping. Increasing the number of zeros trivializes the calculation and the reduction significantly complicates them. Even so, we believe that we can reduce the number of zeros.

Algorithms reducing the number of zeros seem to be difficult, as we mentioned earlier. It is sufficient to consider the case when the number of zeros equals $k-2$. The algorithms reducing the number of zeros will be presented in future articles.

\section{References}

[1] Griffiths P., Harris J., Principles of Algebraic Geometry, New York 1978.

[2] Mumford D., Algebraic Geometry I: Complex Projective Varieties, Springer-Verlag, New York 1975.

[3] Shafarevich I.R., Basic Algebraic Geometry, Springer-Verlag, Berlin, New York 1974.

[4] Wright D., On the Jacobian conjecture, Illinois J. Math. 1981, 25, 3, 423-440.

[5] Van den Essen A., Polynomial Automorphisms and the Jacobian Conjecture, Progress in Mathematics 190, Birkhäuser Verlag, Basel 2000.

[6] Bass H., Connell E.H., Wright D., The Jacobian conjecture: reduction of degree and formal expansion of the inverse, American Mathematical Society. Bulletin. New Series 1982, 7(2), 287-330. 